\title{
ACTIONS OF LOCALLY COMPACT (QUANTUM) GROUPS ON TERNARY RINGS OF OPERATORS, THEIR CROSSED PRODUCTS AND GENERALIZED POISSON BOUNDARIES
}

\author{
PEKKA SALMI AND ADAM SKALSKI
}

\begin{abstract}
Actions of locally compact groups and quantum groups on $\mathrm{W}^{*}$-ternary rings of operators are discussed and related crossed products introduced. The results generalise those for von Neumann algebraic actions with proofs based mostly on passing to the linking von Neumann algebra. They are motivated by the study of fixed point spaces for convolution operators generated by contractive, non-necessarily positive measures, both in the classical and in the quantum context.
\end{abstract}

The notion of a Poisson boundary, related to spaces of harmonic functions in the context of the probability theory, i.e. the fixed points of convolution operators associated to probability measures, has played an important role in the theory of random walks and various aspects of the potential theory for more than 40 years. Starting from the groundbreaking work of Izumi in [Izu, the concept (originally introduced for random walks on $\mathbb{Z}$, but later studied for any locally compact group) was extended also to quantum groups. For the history of further developments we refer to the article $\left[\mathrm{KNR}_{2}\right]$, where the abstract structure of non-commutative Poisson boundaries is studied in detail and connected to the crossed products of von Neumann algebras. The quantum extension shows very clearly that from the modern point of view the construction of the Poisson boundary is a special instance of the Choi-Effros product from the theory of operator algebras granting a von Neumann algebra structure to a space of fixed points of a given unital completely positive map on a von Neumann algebra; this is indeed one of the key observations which led to the current work.

When one considers convolution operators, be it in the classical or the quantum framework, it is natural to analyse not only those associated to probability measures, but also those arising from general signed measures. In general the problem of the study of the fixed points becomes then far more complicated, and even the characterisation of idempotent signed measures (i.e. those whose convolution operators are idempotent maps) remains unknown for non-abelian groups - we refer to NSSS for a longer discussion. The questions become more tractable if one focuses on contractive signed measures. In the classical and the dual to classical context related issues are studied for example in the monograph [ChL]; in the quantum framework the idempotent problem was solved in [NSSS. In both of these works one saw a natural emergence of the $\mathrm{W}^{*}$-TRO structures ( $W^{*}$-ternary rings of operators). This was a motivation for a systematic study of the spaces of fixed points of completely contractive maps which we conduct in this paper.

It turns out that whenever $\mathrm{M}$ is a von Neumann algebra and $P: \mathrm{M} \rightarrow \mathrm{M}$ is a completely contractive normal map, then a Choi-Effros type construction, exploiting the algebraic properties of $P$ established in [You], equips the space Fix $P$ with a unique structure of a $\mathrm{W}^{*}-\mathrm{TRO}$,

2000 Mathematics Subject Classification. Primary 46L65, Secondary 43A05, 47L05.

Key words and phrases. Locally compact quantum group, ternary ring of operators, crossed product. 
which we may call a generalized noncommutative Poisson boundary related to $P$. To understand the structure of the resulting TRO for a contractive convolution operator, one needs to develop also the notion of (quantum) group actions on $\mathrm{W}^{*}$-TROs and analyse appropriate crossed products (in the classical context one can find related work in $\mathrm{Ha}_{2}$ ). Several theorems follow here relatively easily from their von Neumann algebraic counterparts, as each $\mathrm{W}^{*}$-TRO is a corner in its linking von Neumann algebra; some other require certain care, as we mention below.

Once a satisfactory theory of crossed products is developed, it is natural to expect a generalisation of the main result of $\mathrm{KNR}_{2}$, which would show that the generalised noncommutative Poisson boundary for an 'extended' contractive convolution operator $\Theta_{\mu}$ is isomorphic to the crossed product of the analogous boundary for the 'standard' convolution operator $R_{\mu}$ by a natural action of the underlying group. This is indeed what we prove here, but only for classical locally compact groups (the positive case considered in $\mathrm{KNR}_{2}$ yielded the result for general locally compact quantum groups). Here we see an example where the passage from the von Neumann algebra framework to the TRO case is highly non-trivial - very roughly speaking the reason is that the Choi-Effros type construction connects a given concrete initial data (the pair $(\mathrm{M}, P)$ ) with an abstract $\mathrm{W}^{*}$-TRO $\mathrm{X}$ - and the linking von Neumann algebra of $\mathrm{X}$ arises naturally only once we fix a concrete realisation of the latter, which need not be related in any explicit way to the original data.

The plan of the article is as follows: in the first section we recall basic facts regarding the ternary rings of operators, prove a few technical lemmas and connect fixed points of the completely contractive maps to the TROs, introducing the corresponding version of the Choi-Effros product and discussing its basic properties. In Section 2 we develop the notion of actions of locally compact groups on TROs and construct respective crossed products, carefully developing various points of view on this concept. The next section extends the construction to the case of locally compact quantum groups and deals with certain technicalities describing the way in which one can induce actions on TROs arising as fixed point spaces. These are applied in Section 4 to the discussion of the TROs arising from fixed point spaces of contractive convolution operators on locally compact (quantum) groups.

The angled brackets will denote the closed linear span. Hilbert space scalar products will be linear on the right. For a locally compact group $G$ we write $L^{2}(G)$ for the $L^{2}$-space with respect to the left invariant Haar measure; the group von Neumann algebra $\operatorname{VN}(G)$, will be the von Neumann algebra generated by the left regular representation.

Acknowledgement. The work on this article began during our joint Research in Pairs visit with Matthias Neufang and Nico Spronk to the Mathematisches Forschungsinstitut Oberwolfach in August 2012. We are very grateful to MFO for providing ideal research conditions and to both Matthias and Nico for many observations which initiated this work. AS would also like to thank Zhong-Jin Ruan for stimulating discussions on the subject of the paper. AS was partially supported by the NCN (National Centre of Science) grant 2014/14/E/ST1/00525.

\section{1. $\mathrm{W}^{*}$-TERNARY RINGS OF OPERATORS AND FIXED POINTS OF COMPLETELY CONTRACTIVE MAPS}

Recall that a (concrete) TRO, i.e. a ternary ring of operators, $\mathrm{X}$ is a closed subspace of $B(\mathrm{H} ; \mathrm{K})$, where $\mathrm{H}$ and $\mathrm{K}$ are some Hilbert spaces, which is closed under the ternary product: 
$(a, b, c) \mapsto a b^{*} c$. TROs possess natural operator space structure and in fact can be also characterised abstractly, as operator spaces with a ternary product satisfying certain properties, see $\left[\mathrm{NeR}\right.$. To each TRO $\mathrm{X}$ one can associate a $\mathrm{C}^{*}$-algebra $\mathrm{A}_{\mathrm{X}} \subset B(\mathrm{~K} \oplus \mathrm{H})$, called the linking algebra of $\mathbf{X}$. It is explicitly defined as

$$
\mathrm{A}_{\mathrm{X}}:=\left(\begin{array}{cc}
\left\langle\mathrm{XX}^{*}\right\rangle & \mathrm{X} \\
\mathrm{X}^{*} & \left\langle\mathrm{X}^{*} \mathrm{X}\right\rangle
\end{array}\right) \subset B(\mathrm{~K} \oplus \mathrm{H}) .
$$

We always view $X, X^{*}$ and the $C^{*}$-algebras $\left\langle X X^{*}\right\rangle,\left\langle X^{*} X\right\rangle$ as subspaces of $A_{X}$. If $X$ and $Y$ are TROs, then a linear map $\alpha: \mathrm{X} \rightarrow \mathrm{Y}$ is said to be a TRO morphism if it preserves the ternary product. A TRO morphism admits a unique extension to a ${ }^{*}$-homomorphism $\gamma: A_{X} \rightarrow A_{Y}-$ this was proved by M. Hamana in $\mathrm{Ha}_{1}$ (see also [Ha2 and [Zet]) and we will call this map the Hamana extension of $\alpha$. Note that $\gamma$ is defined in a natural way, so for example if $x \in \mathbf{X}$ then $\gamma\left(x x^{*}\right)=\alpha(x) \alpha(x)^{*}$. Further we call a TRO morphism $\alpha: \mathrm{X} \rightarrow \mathrm{Y}$ non-degenerate if the linear spans of $\alpha(\mathrm{X}) \mathrm{Y}^{*} \mathrm{Y}$ and $\alpha(\mathrm{X})^{*} \mathrm{Y}^{*}$ are norm dense respectively in $\mathrm{Y}$ and $\mathrm{Y}^{*}$; in other words the space $\alpha(\mathrm{X})$, which is a sub-TRO of $\mathrm{Y}$ by [Ha1], is a non-degenerate sub-TRO of $\mathrm{Y}$, as defined for example in $\left[\mathrm{SS}_{2}\right]$. Then using the Hamana extensions one can easily show that $\alpha$ is non-degenerate if and only if its Hamana extension $\gamma: A_{X} \rightarrow A_{Y}$ is non-degenerate (as a ${ }^{*}$-homomorphism between $\mathrm{C}^{*}$-algebras) - see Proposition 1.1 of $\left[\mathrm{SS}_{2}\right.$ for this result phrased in terms of sub-TROs.

We say that $\mathrm{X}$ is a $W^{*}$-TRO if it is weak ${ }^{*}$-closed in $B(\mathrm{H} ; \mathrm{K})$. We will usually assume that the TROs we study are non-degenerately represented, i.e. $\langle\mathrm{XH}\rangle=\mathrm{K},\left\langle\mathrm{X}^{*} \mathrm{~K}\right\rangle=\mathrm{H}$. The linking von Neumann algebra associated to $\mathrm{X}$, equal to $\mathrm{A}_{\mathrm{X}}^{\prime \prime}$, will be denoted by $\mathrm{R}_{\mathrm{X}}$, so that

$$
\mathrm{R}_{\mathrm{X}}:=\left(\begin{array}{cc}
\left\langle\mathrm{XX}^{*}\right\rangle^{\prime \prime} & \mathrm{X} \\
\mathrm{X}^{*} & \left\langle\mathrm{X}^{*} \mathrm{X}\right\rangle^{\prime \prime}
\end{array}\right) \subset B(\mathrm{~K} \oplus \mathrm{H}) \text {. }
$$

For a TRO morphism between $\mathrm{W}^{*}$-TROs non-degeneracy will mean that the linear spans of the spaces introduced in the paragraph above are weak*-dense in the respective TROs. The predual of a $\mathrm{W}^{*}$-TRO $\mathrm{X}$ will be denoted by $\mathrm{X}_{*}$; it is not difficult to see that $\mathrm{X}_{*}=\left\{\left.\omega\right|_{\mathrm{X}}: \omega \in\right.$ $\left.\left(\mathrm{R}_{\mathrm{X}}\right)_{*}\right\}$.

There is also an abstract characterisation of TROs and $\mathrm{W}^{*}$-TROs due to Zettl [Zet], which we now recall. An abstract TRO is a Banach space $\mathrm{X}$ equipped with a ternary operation

$$
\{\cdot, \cdot, \cdot\}: \mathrm{X} \times \mathrm{X} \times \mathrm{X} \rightarrow \mathrm{X}
$$

such that the following conditions hold $(x, y, z, u, v \in \mathrm{X})$ :

(1) the operation is linear in the first and the third variable and conjugate linear in the second,

(2) $\{\{x, y, z\}, u, v\}=\{x,\{u, z, y\}, v\}=\{x, y,\{z, u, v\}\}$;

(3) $\|\{x, y, z\}\| \leq\|x\|\|y\|\|z\|$;

(4) $\|\{x, x, x\}\|=\|x\|^{3}$.

An abstract $W^{*}-T R O$ is an abstract TRO that is a dual Banach space. Zettl [Zet] proved that these abstractly defined objects have concrete representations as TROs and $\mathrm{W}^{*}$-TROs, respectively.

The next result is a $\mathrm{W}^{*}$-version of the fact due to Hamana regarding images of TROs, observed in [BLM].

Lemma 1.1. If $\mathrm{X}$ and $\mathrm{Y}$ are $W^{*}$-TROs, and $\alpha: \mathrm{X} \rightarrow \mathrm{Y}$ is a normal TRO morphism, then $\alpha(\mathbf{X})$ is a $W^{*}-T R O$. 
Proof. This is proved in Section 8.5.18 in [BLM]. The main idea is as follows: the kernel of $\alpha$ is a sub-TRO of $\mathrm{X}$. Thus it can be written as $f \mathbf{X}$, where $f$ is a central projection in the von Neumann algebra $\left\langle\mathrm{XX}^{*}\right\rangle^{\prime \prime}$. The morphism $\alpha^{\prime}:(1-f) \mathrm{X} \rightarrow \mathrm{Y}$ given by the restriction of $\alpha$ is then easily seen to be injective; moreover $\alpha^{\prime}(\mathbf{X})=\alpha(\mathbf{X})$. Then we deduce that the unit ball of $\alpha^{\prime}(\mathbf{X})$ is the image of the unit ball of $\mathbf{X}$, hence it is weak*-compact (so in particular weak *-closed).

Proposition 3.1 of $\mathrm{SS}_{2}$ shows that a TRO morphism $\alpha: \mathrm{X} \rightarrow \mathrm{Y}$ is non-degenerate if and only if the $\mathrm{W}^{*}$-TRO $\alpha(\mathrm{X})$ is non-degenerately represented. The last lemma can be used to note that Hamana extensions can be considered also in the $\mathrm{W}^{*}$-category and moreover have the expected properties.

Proposition 1.2. Let $\mathrm{X}$ and $\mathrm{Y}$ be $W^{*}$-TROs and let $\alpha: \mathrm{X} \rightarrow \mathrm{Y}$ be a normal TRO morphism. Then there exists a unique normal ${ }^{*}$-homomorphism $\beta: \mathrm{R}_{\mathrm{X}} \rightarrow \mathrm{R}_{\mathrm{Y}}$ such that

$$
\beta\left(\begin{array}{ll}
0 & x \\
0 & 0
\end{array}\right)=\left(\begin{array}{cc}
0 & \alpha(x) \\
0 & 0
\end{array}\right), \quad x \in \mathrm{X} .
$$

Moreover $\left.\beta\right|_{\mathrm{Ax}_{\mathrm{x}}}$ is the Hamana extension discussed above, the extension construction preserves the composition, and moreover

(i) $\alpha$ is injective if and only if $\beta$ is injective;

(ii) $\alpha$ is non-degenerate if and only if $\beta$ is unital.

Proof. There are at least two ways to see the first statement (the rest is relatively easy). In the first step one observes that we can assume that $\alpha$ is surjective, using the last lemma (indeed, if $\mathbf{Z}=\alpha(\mathbf{X})$, then $\mathbf{R}_{\mathbf{Z}}$ is a von Neumann subalgebra of $\mathbf{R}_{\mathbf{Y}}$ ).

Now we can either proceed directly, as in $\left[\mathrm{SS}_{2}\right.$, using non-degeneracy, or first pass to the situation where $\alpha$ is isometric, quotienting out its kernel (this leads to another $\mathrm{W}^{*}$-TRO, as follows from $\left[\mathrm{Ha}_{1}\right]$ ) and then use the proof of Corollary $3.4 \mathrm{in}$ [Sol]. The reason we cannot use this corollary directly is that we need to verify that the map obtained there (or in fact rather via Theorem 2.1 of that paper) coincides with the weak*-continuous extension of the Hamana extension. This however can be checked directly, following the arguments in Corollary 3.4 and Lemma 2.5 of [Sol].

Injectivity of $\alpha$ (respectively, $\beta$ ) is equivalent to $\alpha$ (respectively, $\beta$ ) being isometric; thus Sol implies that injectivity of $\alpha$ is equivalent to that of $\beta$.

For a $\mathrm{W}^{*}$-TRO $\mathrm{X} \subset B(\mathrm{H} ; \mathrm{K})$ it is elementary to check that $\mathrm{X}$ is non-degenerately represented if and only if $\mathrm{R}_{\mathbf{X}}$ contains the unit of $B(\mathrm{~K} \oplus \mathrm{H})$. This together with the comments before the proposition implies the last statement.

Note that in the situation above by boundedness and normality of the maps in question, we have the following consequence of the algebraic form of Hamana extensions (in which we view both $\mathrm{X}$ and $\left\langle\mathrm{XX}^{*}\right\rangle^{\prime \prime}$ as subspaces of $\left.\mathrm{R}_{\mathrm{X}}\right)$ :

$$
\beta(z) \alpha(x)=\alpha(z x), \quad x \in \mathbf{X}, z \in\left\langle\mathbf{X X}^{*}\right\rangle^{\prime \prime} .
$$

If $\mathrm{X}$ happens to be a von Neumann algebra, $\mathrm{R}_{\mathrm{X}} \cong M_{2}(\mathrm{X})$; if $\mathrm{Y}$ is another von Neumann algebra and we assume that $\alpha: \mathrm{X} \rightarrow \mathrm{Y}$ is a ${ }^{*}$-homomorphism, then $\beta$ is the usual matrix lifting of $\alpha$. Finally we note an easy observation which will be useful later.

Corollary 1.3. Let $\mathrm{X}$ and $\mathrm{Y}$ be $W^{*}$-TROs and let $\beta: \mathrm{R}_{\mathrm{X}} \rightarrow \mathrm{R}_{\mathrm{Y}}$ be a normal ${ }^{*}$-homomorphism. Then $\beta$ is the Hamana extension of a normal TRO morphism between $\mathrm{X}$ and $\mathrm{Y}$ if and only 
if $\beta(\mathrm{X}) \subset \mathrm{Y}$. If $\mathrm{X}$ and $\mathrm{Y}$ are respectively non-degenerately represented in $B\left(\mathrm{H}_{1} ; \mathrm{K}_{1}\right)$ and in $B\left(\mathrm{H}_{2} ; \mathrm{K}_{2}\right)$, then the conditions above are equivalent to the equality

$$
P_{\mathrm{K}_{2}} \beta\left(P_{\mathrm{K}_{1}} x P_{\mathrm{H}_{1}}\right) P_{\mathrm{H}_{2}}=\beta\left(P_{\mathrm{K}_{1}} x P_{\mathrm{H}_{1}}\right)
$$

being valid for all $x \in \mathrm{R}_{\mathrm{X}}$.

Proof. A simple calculation shows that if $\beta(\mathrm{X}) \subset \mathrm{Y}$, then $\left.\beta\right|_{\mathrm{X}}$ is a TRO morphism. Then the equivalence follows from the uniqueness of Hamana extensions and the second statement is an easy consequence of the definitions of $R_{X}$ and $R_{Y}$.

Given two $\mathrm{W}^{*}$-TROs $\mathrm{X} \subset B\left(\mathrm{H}_{1} ; \mathrm{K}_{1}\right)$ and $\mathrm{Y} \subset B\left(\mathrm{H}_{2} ; \mathrm{K}_{2}\right)$, we can naturally consider the $\mathrm{W}^{*}$-TRO $\mathrm{X} \bar{\otimes} \mathrm{Y}$ defined as the weak ${ }^{*}$ closure of the algebraic tensor product $\mathrm{X} \odot \mathrm{Y} \subset B\left(\mathrm{H}_{1} \otimes\right.$ $\left.\mathrm{H}_{2} ; \mathrm{K}_{1} \otimes \mathrm{K}_{2}\right)$. The fact that it is closed under the ternary product can be easily checked. Note that if $M$ is a von Neumann algebra, then we have a natural identification of $R_{X \bar{X}} M$ with $\mathrm{R}_{\mathrm{X}} \bar{\otimes} \mathrm{M}$; this will be of use later.

Similarly note for the future use that if $\mathrm{Z} \subset B(\mathrm{H})$ is a weak ${ }^{*}$-closed subalgebra and $P \in \mathrm{Z}$ is a projection, then $P \mathrm{Z}$ is weak ${ }^{*}$-closed. This implies that if say $Q \in \mathrm{Z}$ is another projection and $\mathrm{W} \subset B(\mathrm{~K})$ is a weak* ${ }^{*}$-closed subalgebra, then

$$
P \mathrm{Z} Q \bar{\otimes} \mathrm{W}=\left(P \otimes I_{\mathrm{K}}\right)(\mathrm{Z} \bar{\otimes} \mathrm{W})\left(Q \otimes I_{\mathrm{K}}\right) .
$$

As usual, $\mathbf{Z} \bar{\otimes} \mathbf{W}$ denotes the weak* closure of algebraic tensor product $\mathrm{Z} \odot \mathrm{W}$ inside $B(\mathrm{H}) \bar{\otimes} B(\mathrm{~K})$.

Finally recall (for example from Chapter 7 of [ER 1 ) that if $X$ and $Y$ are dual operator spaces, then their Fubini tensor product $\mathrm{X} \otimes_{F} \mathrm{Y}$ is defined abstractly as the operator space dual of $\mathrm{X}_{*} \widehat{\otimes} \mathrm{Y}_{*}$; if $\mathrm{X}$ and $\mathrm{Y}$ are weak ${ }^{*}$-closed subspaces of say $B(\mathrm{H})$ and $B(\mathrm{~K})$, then $\mathrm{X} \otimes_{F} \mathrm{Y}$ can be realised as

$$
\left\{u \in B(\mathrm{H}) \bar{\otimes} B(\mathrm{~K}):(\omega \otimes \mathrm{id}) u \in \mathrm{Y} \text { and }(\mathrm{id} \otimes \sigma) u \in \mathrm{X} \text { for every } \omega \in B(\mathrm{H})_{*}, \sigma \in B(\mathrm{~K})_{*}\right\},
$$

Clearly, $\mathrm{X} \bar{\otimes} \mathrm{Y}$ is contained in $\mathrm{X} \otimes_{F} \mathrm{Y}$.

Lemma 1.4. Let $\mathrm{X}$ and $\mathrm{Y}$ be dual operator spaces that are weak ${ }^{*}$ completely contractively complemented in von Neumann algebras (note that in particular $W^{*}-$ TROs satisfy these assumptions). Then the natural weak*-continuous completely isometric embedding $\mathrm{X} \bar{\otimes} \mathrm{Y} \hookrightarrow \mathrm{X} \otimes_{F} \mathrm{Y}$ is in fact an isomorphism.

Proof. By the assumptions there are von Neumann algebras $R_{\mathrm{X}}$ and $R_{\mathrm{Y}}$ containing $\mathrm{X}$ and $\mathrm{Y}$, respectively, and normal completely contractive projections $P_{\mathrm{X}}: R_{\mathrm{X}} \rightarrow \mathrm{X}$ and $P_{\mathrm{Y}}: R_{\mathrm{Y}} \rightarrow \mathrm{Y}$. The algebraic tensor product $P_{\mathbf{X}} \odot P_{Y}$ extends uniquely to a normal map $P_{\mathbf{X}} \otimes P_{Y}$ from $R_{\mathrm{X}} \bar{\otimes} R_{\mathrm{Y}}=R_{\mathrm{X}} \otimes_{F} R_{\mathrm{Y}}$ to $\mathrm{X} \otimes_{F} \mathrm{Y}$ (see Chapter 7 of $\left[\mathrm{ER}_{1}\right.$ and Proposition 4.3 of $\left[\mathrm{ER}_{2}\right]$ ). As $\mathrm{X} \otimes_{F} \mathrm{Y} \subset R_{\mathrm{X}} \bar{\otimes} R_{\mathrm{Y}}$, the uniqueness of extensions implies that $P_{\mathrm{X}} \otimes P_{\mathrm{Y}}$ is the identity map when restricted to $\mathbf{X} \otimes_{F} \mathrm{Y}$. Let $u \in \mathrm{X} \otimes_{F} \mathrm{Y}$ and let $\left(u_{i}\right)_{i \in \mathcal{I}}$ be a net in the algebraic tensor product $R_{\mathbf{X}} \odot R_{Y}$ that converges to $u$ in the weak* topology. Then

$$
u=\left(P_{\mathbf{X}} \otimes P_{\mathbf{Y}}\right)(u)=\mathrm{w}^{*}-\lim _{i \in \mathcal{I}}\left(P_{\mathbf{X}} \odot P_{\mathbf{Y}}\right)\left(u_{i}\right) \in \mathbf{X} \bar{\otimes} \mathbf{Y} .
$$

Consider then a TRO morphism $\alpha: \mathrm{X} \rightarrow \mathrm{Y}$. It follows from $\mathrm{Ha}_{1}$ that $\alpha$ is completely contractive. Moreover, Proposition 1.1 of $\mathrm{Ha}_{2}$ implies that if $Z$ is another $\mathrm{W}^{*}$-TRO, then the map id $\mathrm{Z} \otimes \alpha$ extends uniquely to a completely contraction from $\mathbf{Z} \otimes_{F} \mathbf{X}$ to $\mathbf{Z} \otimes_{F} \mathrm{Y}$ - this does not require that the original map is normal. If $\alpha$ is in addition normal, the resulting extension is also normal, as follows for example from the identification of the predual of 
the Fubini tensor product as the projective tensor product of the preduals of the individual factors. So when $\alpha$ is normal, we can view $\mathrm{id}_{\mathbf{Z}} \otimes \alpha$ as a normal TRO morphism from $\mathbf{Z} \bar{\otimes} \mathrm{X}$ to $Z \bar{\otimes} Y$. If we want to stress that we are working with a not necessarily normal extension we will write $\operatorname{idz} \otimes_{F} \alpha$.

The celebrated Choi-Effros construction equips a fixed point space of a completely positive map with a $\mathrm{C}^{*}$-algebra structure. Below we present an analogous result for completely contractive maps and TRO structures.

The first proposition is essentially a theorem of Youngson [You] (see also Theorem 4.4.9 in BLM] $)$.

Proposition 1.5. Let $\mathrm{A}$ be a $C^{*}$-algebra and let $P: \mathrm{A} \rightarrow \mathrm{A}$ be a completely contractive projection. Then $\widetilde{\mathrm{X}}:=P(\mathrm{~A})$ possesses a TRO structure, with the product given by the formula

$$
\{a, b, c\}:=P\left(a b^{*} c\right), \quad a, b, c \in \widetilde{\mathrm{X}} .
$$

Denote the resulting TRO by $\mathrm{X}$. Then the identity map $\iota: \widetilde{\mathrm{X}} \rightarrow \mathrm{X}$ is a completely isometric isomorphism (where $\widetilde{\mathbf{X}}$ inherits the operator space structure from $\mathbf{A}$ and $\mathbf{X}$ is an operator space as a TRO). If $\mathrm{A}$ is a von Neumann algebra and $\widetilde{\mathrm{X}}$ happens to be weak ${ }^{*}$-closed, then $\mathrm{X}$ is a $W^{*}-T R O$ and $\iota: \widetilde{\mathrm{X}} \rightarrow \mathrm{X}$ is in addition a homeomorphism for weak $k^{*}$ topologies.

Proof. The fact that the displayed formula defines a TRO structure (with the norm induced from A) is the Theorem of [You] (p. 508) - it follows also from the abstract description due to Zettl mentioned earlier. The map $\iota$ is thus an isometry. Applying the same construction to $P^{(n)}: M_{n}(\mathrm{~A}) \rightarrow M_{n}(\mathrm{~A})$ gives a TRO based on $M_{n}(\widetilde{\mathrm{X}})$ and this TRO is naturally isomorphic to $M_{n}(\mathrm{X})$. Proposition 2.1 of $\mathrm{Ha}_{1}$ implies that this isomorphism is an isometry. Thus $\iota$ is in fact a complete isometry. The second part follows from the uniqueness of a predual of a $\mathrm{W}^{*}$-TRO (Proposition 2.4 of [EOR]).

Theorem 1.6. Let $\mathrm{M}$ be a von Neumann algebra and let $P: \mathrm{M} \rightarrow \mathrm{M}$ be a completely contractive normal map. Consider the space Fix $P=\{x \in \mathrm{M}: P x=x\}$. Then Fix $P$ is a weak $k^{*}$-closed subspace of $\mathrm{M}$, so in particular a dual operator space. It possesses a unique ternary product which makes it a $W^{*}-T R O$. It is explicitly given by the formula

$$
\{a, b, c\}:=\widetilde{P}_{\beta}\left(a b^{*} c\right), \quad a, b, c \in \operatorname{Fix} P,
$$

where $\beta$ is a fixed free ultrafilter,

$$
\widetilde{P}_{\beta}(x)=\beta-\lim _{n \in \mathbb{N}} \frac{1}{n} \sum_{k=0}^{n-1} P^{k}(x), \quad x \in \mathrm{M},
$$

and the last limit is understood in a weak $k^{*}$ topology.

Proof. To show the existence of the ternary product described above it suffices to verify that $P_{\beta}: \mathrm{M} \rightarrow \mathrm{M}$ is a completely contractive projection onto Fix $P$. The fact that $P_{\beta}$ is a projection onto Fix $P$ follows by standard Cesàro limit arguments; the (complete) contractivity of $P_{\beta}$ follows from the analogous property of $P$ and the fact that a weak ${ }^{*}$ limit of contractions is a contraction. The uniqueness of the ternary product follows once again from Proposition 2.1 of $\mathrm{Ha}_{1}$.

Remark 1.7. Let us stress that Proposition 1.5 implies in particular that the $\mathrm{W}^{*}-\mathrm{TRO}$ structure of Fix $P$ does not depend on the choice of the ultrafilter in the above proof (although the map $\widetilde{P}_{\beta}$ may well do). 
The following result is an abstract extension of Proposition 3.3.1 of [ChL], the proof is essentially the same.

Proposition 1.8. Suppose that the assumptions of Theorem 1.6 hold. If there exists a normal

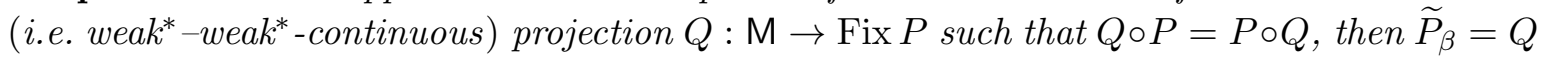
for any free ultrafilter $\beta$.

Proof. Recall that a normal projection is necessarily bounded. Thus we have for each $x \in \mathrm{M}$ (and a free ultrafilter $\beta$ )

$$
\begin{aligned}
\widetilde{P}_{\beta}(x) & =Q \widetilde{P}_{\beta}(x)=Q\left(\beta-\lim _{n \in \mathbb{N}} \frac{1}{n} \sum_{k=0}^{n-1} P^{k}(x)\right) \\
& =\beta-\lim _{n \in \mathbb{N}} Q\left(\frac{1}{n} \sum_{k=0}^{n-1} P^{k}(x)\right)=\beta-\lim _{n \in \mathbb{N}} \frac{1}{n} \sum_{k=0}^{n-1} P^{k}(Q x)=Q x .
\end{aligned}
$$

\section{ACtions of LOCALLY COMPACT GROUPS ON W*-TROS AND RESUlting CROSSED PRODUCTS}

In this section we discuss actions of locally compact groups on $\mathrm{W}^{*}$-TROs and the associated crossed products. Analogous study in the operator space context was undertaken in $\mathrm{Ha}_{2}$; we will comment on some specific analogies at the end of this section.

Definition 2.1. Let $G$ be a locally compact group, and let $\mathrm{X}$ be a $\mathrm{W}^{*}-\mathrm{TRO}$. Denote by Aut $(X)$ the set of all normal automorphisms of X, i.e. normal bijective TRO morphisms from $\mathrm{X}$ onto itself. A (continuous) action of $G$ on $\mathrm{X}$ is a homomorphism $\alpha: G \rightarrow \operatorname{Aut}(\mathrm{X})$ such that for each $x \in \mathrm{X}$ the map $\alpha^{x}: G \rightarrow \mathrm{X}$ defined by

$$
\alpha^{x}(s)=(\alpha(s))(x), \quad s \in G,
$$

is weak*-continuous. We shall write $\alpha_{s}=\alpha(s)$ for $s \in G$.

The continuity condition above has several equivalent formulations which can be deduced from Sections 13.4 and 13.5 of $[\mathrm{Str}]$. We record one of them in the following proposition.

Proposition 2.2. Let $G$ be a locally compact group, and let $\mathrm{X}$ be a $W^{*}$-TRO. A homomorphism $\alpha: G \rightarrow \operatorname{Aut}(\mathrm{X})$ is a continuous action of $G$ on $\mathbf{X}$ if and only if the map $G \times \mathrm{X}_{*} \ni(s, \varphi) \mapsto \varphi \circ \alpha_{s} \in \mathrm{X}_{*}$ is norm-continuous.

We are ready to connect the action of $G$ on $\mathrm{X}$ with the action on $\mathrm{R}_{\mathbf{X}}$.

Theorem 2.3. Let $\alpha$ be an action of a locally compact group $G$ on a $W^{*}-T R O X$. Then it possesses a unique extension to an action of $G$ on $\mathrm{R}_{\mathrm{X}}$.

Proof. First fix $g \in G$ and extend $\alpha_{g} \in \operatorname{Aut}(\mathrm{X})$ to a normal automorphism $\beta_{g} \in \operatorname{Aut}\left(\mathrm{R}_{\mathrm{X}}\right)$ via Proposition 1.2.

The uniqueness of the extensions implies that the resulting family $\left\{\beta_{g}: g \in G\right\}$ defines a homomorphism $\beta: G \rightarrow \operatorname{Aut}\left(\mathrm{R}_{\mathrm{X}}\right)$. It remains to check that it satisfies the continuity requirement. We do it separately for each corner of the map $\beta$, presenting the argument only for the upper left corner. 
Take $z \in\left\langle\mathrm{XX}^{*}\right\rangle^{\prime \prime}$ and consider the map $\beta^{z}: G \rightarrow \mathrm{R}_{\mathbf{X}}$. We need to show it is weak*continuous. As all the maps in question are contractive and we may assume that $\mathrm{X}$ is nondegenerately represented in $B(\mathrm{H} ; \mathrm{K})$, it suffices to check that for all $\xi \in \mathrm{K}, x \in \mathrm{X}, \eta \in \mathrm{H}$, and a net of elements $\left(s_{i}\right)_{i \in \mathcal{I}}$ of $G$ converging to $e \in G$, we have

$$
\left\langle\xi, \beta_{s_{i}}(z) x \eta\right\rangle \stackrel{i \in \mathcal{I}}{\longrightarrow}\langle\xi, z x \eta\rangle .
$$

Note that, by (1.1),

$$
\left\langle\xi, \beta_{s_{i}}(z) x \eta\right\rangle=\left\langle\xi, \beta_{s_{i}}(z) \alpha_{s_{i}}\left(\alpha_{s_{i}^{-1}}(x)\right) \eta\right\rangle=\left\langle\xi, \alpha_{s_{i}}\left(z \alpha_{s_{i}^{-1}}(x)\right) \eta\right\rangle=\omega_{\xi, \eta} \circ \alpha_{s_{i}}\left(z \alpha_{s_{i}^{-1}}(x)\right),
$$

so putting $\omega:=\omega_{\xi, \eta}$ we obtain

$$
\left\langle\xi, \beta_{s_{i}}(z) x \eta\right\rangle-\langle\xi, z x \eta\rangle=\left(\omega \circ \alpha_{s_{i}}-\omega\right)\left(z \alpha_{s_{i}^{-1}}(x)\right)+\omega\left(z \alpha_{s_{i}^{-1}}(x)-z x\right) .
$$

Applying Proposition [2.2, we see that the upper left corner of $\beta^{z}$ is weak*-continuous. The remaining parts of the proof follow analogously.

For an action $\alpha$ of $G$ on a $\mathrm{W}^{*}$-TRO $\mathrm{X}$ we define the fixed point space Fix $\alpha$ as

$$
\text { Fix } \alpha=\left\{x \in \mathrm{X}: \forall_{g \in G} \alpha_{g}(x)=x\right\} \text {. }
$$

It is clear that Fix $\alpha$ is a $\mathrm{W}^{*}$-sub-TRO of $\mathrm{X}$.

Corollary 2.4. Assume that $\mathrm{X}$ is a $W^{*}$-TRO that is non-degenerately represented in some $B(\mathrm{H} ; \mathrm{K}), \alpha$ is an action of $G$ on $\mathrm{X}$ and $\beta$ is an action of $G$ on $\mathrm{R}_{\mathrm{X}}$ introduced in Theorem 2.3. Then Fix $\alpha=P_{\mathrm{K}}(\operatorname{Fix} \beta) P_{\mathrm{H}}$.

Proof. Let $x \in \mathrm{X}, g \in G$. If $\alpha_{g}(x)=x$ then we also have $\beta_{g}(x)=x$, and naturally $P_{\mathrm{K}} x P_{\mathrm{H}}=x$, which proves the inclusion ' $C$ ' in the desired equality. On the other hand if $x=P_{\mathrm{K}} z P_{\mathrm{H}}$ for some $z \in \operatorname{Fix} \beta$ then

$$
\alpha_{g}(x)=\beta_{g}(x)=\beta_{g}\left(P_{\mathrm{K}} z P_{\mathrm{H}}\right)=\beta_{g}\left(P_{\mathrm{K}}\right) \beta_{g}(z) \beta_{g}\left(P_{\mathrm{H}}\right)=P_{\mathrm{K}} z P_{\mathrm{H}}=x,
$$

where we used the fact that $\beta_{g}$ is a homomorphism and that (by construction) it preserves the projections $P_{\mathrm{K}}$ and $P_{\mathrm{H}}$.

We now discuss the connection of pointwise actions defined above with their integrated incarnations. The interplay between the two plays a crucial role in $\mathrm{Ha}_{2}$ - the situation studied there is however subtler, as the $\mathrm{W}^{*}$-context (as opposed to the $\mathrm{C}^{*}$-problems studied by Hamana) and presence of linking von Neumann algebras leads to certain simplifications. Recall that if $G$ is a locally compact group, then $L^{\infty}(G)$ admits a natural coproduct (see also Section 3) $\Delta: L^{\infty}(G) \rightarrow L^{\infty}(G) \bar{\otimes} L^{\infty}(G)$, defined via the isomorphism $L^{\infty}(G) \bar{\otimes} L^{\infty}(G) \cong$ $L^{\infty}(G \times G)$ and the formula

$$
\Delta(f)(g, h)=f(g h), \quad f \in L^{\infty}(G), g, h \in G .
$$

Theorem 2.5. Suppose that $\alpha: G \rightarrow \operatorname{Aut}(\mathrm{X})$ is an action of a locally compact group $G$ on a $W^{*}$-TRO $\mathrm{X}$. Then there exists a unique map $\pi_{\alpha}: \mathrm{X} \rightarrow L^{\infty}(G) \bar{\otimes} \mathrm{X}$ such that for each $f \in L^{1}(G), \phi \in \mathrm{X}_{*}$ and $x \in \mathrm{X}$ we have

$$
(f \otimes \phi)\left(\pi_{\alpha}(x)\right)=\int_{G} f(g) \phi\left(\alpha_{g^{-1}}(x)\right) d g .
$$


Moreover, if we write $\gamma:=\pi_{\alpha}$, then $\gamma$ is an injective, normal, non-degenerate TRO morphism such that

$$
(\Delta \otimes \mathrm{idx}) \circ \gamma=\left(\mathrm{id}_{L^{\infty}(G)} \otimes \mathrm{idx}\right) \circ \gamma .
$$

Conversely, if $\gamma: \mathrm{X} \rightarrow L^{\infty}(G) \bar{\otimes} \mathrm{X}$ is an injective, normal, non-degenerate TRO morphism satisfying (2.2), then there exists a unique action $\alpha$ of $G$ on $\mathrm{X}$ such that $\gamma=\pi_{\alpha}$.

Proof. We may assume that $\mathrm{X}$ is non-degenerately represented in $B(\mathrm{H} ; \mathrm{K})$.

Assume first that we are given an action $\alpha: G \rightarrow \operatorname{Aut}(\mathrm{X})$ and extend it pointwise, using Theorem 2.3, to a continuous action $\beta: G \rightarrow \operatorname{Aut}\left(\mathrm{R}_{\mathrm{X}}\right)$. Discussion in Section 18.6 of [Str] implies that there exists an injective, normal, unital ${ }^{*}$-homomorphism $\pi_{\beta}: \mathrm{R}_{\mathrm{X}} \rightarrow L^{\infty}(G) \otimes \mathrm{R}_{\mathrm{X}}$ such that for each $f \in L^{1}(G), \phi \in\left(\mathrm{R}_{\mathrm{X}}\right)_{*}$ and $z \in \mathrm{R}_{\mathrm{X}}$ we have

$$
(f \otimes \phi)\left(\pi_{\beta}(z)\right)=\int_{G} f(g) \phi\left(\beta_{g^{-1}}(z)\right) d g
$$

and $(\Delta \otimes \mathrm{id}) \circ \pi_{\beta}=\left(\mathrm{id} \otimes \pi_{\beta}\right) \circ \pi_{\beta}$ (note that our formulas are formally different from Stratila's: the difference is however only in the fact that we choose to work with maps taking values in $L^{\infty}(G) \bar{\otimes} \mathrm{R}_{\mathrm{X}}$, and not in $\mathrm{R}_{\mathrm{X}} \bar{\otimes} L^{\infty}(G)$, which allows us to work with the standard coproduct of $L^{\infty}(G)$, and not with the opposite one as it is in [Str] . Consider the map $\pi_{\beta} \mid \mathrm{x}$. We want to show that it takes values in $\mathrm{Y}:=L^{\infty}(G) \bar{\otimes} \mathrm{X}$. Considerations before Lemma 1.4 imply that the latter space is a $\mathrm{W}^{*}$-TRO equal to $\left(I_{L^{2}(G)} \otimes P_{\mathrm{K}}\right)\left(L^{\infty}(G) \bar{\otimes} \mathrm{R}_{\mathrm{X}}\right)\left(I_{L^{2}(G)} \otimes P_{\mathrm{H}}\right)$; moreover $\mathrm{R}_{\mathrm{Y}}=L^{\infty}(G) \bar{\otimes} \mathrm{R}$. Let then $x \in \mathrm{X}$. It suffices to show that for any $f \in L^{1}(G), \phi \in\left(\mathrm{R}_{\mathrm{X}}\right)_{*}$ we have

$$
(f \otimes \phi)\left(\pi_{\beta}(x)\right)=(f \otimes \phi)\left(\left(I_{L^{2}(G)} \otimes P_{\mathrm{K}}\right) \pi_{\beta}(x)\left(I_{L^{2}(G)} \otimes P_{\mathrm{H}}\right)\right) .
$$

This however follows immediately from (2.3) once we note that $\beta_{g}(x)=\alpha_{g}(x)$ for all $g \in G$ and that $y \mapsto \phi\left(P_{\mathrm{K}} y P_{\mathrm{H}}\right)$ is a normal functional on $\mathrm{R}_{\mathrm{X}}$. Thus we showed that $\gamma:=\pi_{\beta} \mid \mathrm{x}$ maps $\mathrm{X}$ into $\mathrm{Y}$. It is an injective, normal TRO morphism satisfying (2.2) (as a restriction of an injective, normal *-homomorphism satisfying (2.2) ). By the uniqueness of Hamana extensions and the above identification of $R_{Y}$ we deduce that $\pi_{\beta}$ is the Hamana extension of $\gamma$, so that non-degeneracy of $\gamma$ follows from unitality of $\pi_{\beta}$ via Proposition 1.2 .

Assume now that $\gamma: \mathrm{X} \rightarrow L^{\infty}(G) \bar{\otimes} \mathrm{X}$ is an injective, normal, non-degenerate TRO morphism satisfying the action equation (2.2). Again write $\mathrm{Y}=L^{\infty}(G) \bar{\otimes} \mathrm{X}$ and let $\pi: \mathrm{R}_{\mathrm{X}} \rightarrow$ $\mathrm{R}_{\mathrm{Y}}=L^{\infty}(G) \bar{\otimes} \mathrm{R}_{\mathrm{X}}$ denote the Hamana extension of $\gamma$. Proposition 1.2 implies that $\pi$ is a unital, injective normal *-homomorphism. Normality of $\pi$ and $\Delta$ implies that it suffices to check the validity of the action equation with $\gamma$ replaced by $\pi$ on a weak ${ }^{*}$-dense subset; this follows in turn from the computations of the type $(x, z \in \mathrm{X})$ :

$$
\begin{aligned}
\left(\Delta \otimes \mathrm{idx}_{\mathrm{X}}\right)\left(\pi\left(x z^{*}\right)\right) & =\left(\Delta \otimes \mathrm{idx}_{\mathrm{X}}\right)\left(\gamma(x) \gamma(z)^{*}\right)=\left(\Delta \otimes \mathrm{idx}_{\mathrm{X}}\right)(\gamma(x))\left(\Delta \otimes \mathrm{idx}_{\mathbf{X}}\right)(\gamma(z))^{*} \\
& =\left(\operatorname{id}_{L^{\infty}(G)} \otimes \gamma\right)(\gamma(x))\left(\operatorname{id}_{L^{\infty}(G)} \otimes \gamma\right)(\gamma(z))^{*}=\left(\operatorname{id}_{L^{\infty}(G)} \otimes \pi\right)\left(\gamma(x) \gamma(z)^{*}\right) \\
& =\left(\operatorname{id}_{L^{\infty}(G)} \otimes \pi\right)\left(\pi\left(x z^{*}\right)\right) .
\end{aligned}
$$

The Proposition in Section 18.6 of [Str] (or rather its left version) implies that there exists an action $\beta: G \rightarrow \operatorname{Aut}\left(\mathrm{R}_{\mathrm{X}}\right)$ such that $\pi=\pi_{\beta}$, where $\pi_{\beta}$ is defined via formula (2.3). It remains to show that for each $g \in G$ the map $\alpha_{g}:=\beta_{g} \mid \times$ takes values in $\mathrm{X}$, as then it will be easy to check that the family $\left(\alpha_{g}\right)_{g \in G}$ defines an action of $G$ on $\mathbf{X}$ and that $\gamma$ arises from this action via the formulas given in the theorem. Fix then $x \in \mathrm{X}$ and define $z_{g}=\beta_{g^{-1}}(x)$ for each $g \in G$. 
Then $z: G \rightarrow \mathrm{R}_{\mathrm{X}}$ is a weak*-continuous function and we know that for all $f \in L^{1}(G)$ and all $\phi \in\left(\mathrm{R}_{\mathrm{X}}\right)_{*}$ such that $\phi(\mathrm{X})=\{0\}$ we have

$$
\int_{G} f(g) \phi\left(z_{g}\right) d g=0 .
$$

But then we deduce immediately that $\phi\left(z_{g}\right)$ is 0 for almost all $g \in G$, and as it is a continuous function it must actually be 0 everywhere. This in turn means that $z_{g} \in \mathrm{X}$ for all $g \in G$, which ends the proof.

The next proposition is also familiar from the von Neumann algebraic context.

Proposition 2.6. Suppose that $\alpha: G \rightarrow \operatorname{Aut}(\mathrm{X})$ is an action of a locally compact group $G$ on a $W^{*}-T R O X$ and assume that $\mathrm{X}$ is non-degenerately represented in $B(\mathrm{H} ; \mathrm{K})$. Then the map $\pi_{\alpha}$ introduced in Theorem 2.5 may be viewed as a faithful representation of $\mathbf{X}$ in $B\left(L^{2}(G ; \mathrm{H}) ; L^{2}(G ; \mathrm{K})\right)$, and we have for all $x \in \mathrm{X}$ and $\zeta \in L^{2}(G ; \mathrm{H})$

$$
\left(\pi_{\alpha}(x)(\zeta)\right)(g)=\alpha_{g^{-1}}(x) \zeta(g) \quad \text { for almost every } g \in G \text {. }
$$

Proof. The fact that $\pi_{\alpha}$ can be viewed as a faithful representation of $\mathrm{X}$ in $B\left(L^{2}(G ; \mathrm{H}) ; L^{2}(G ; \mathrm{K})\right)$ follows from Theorem 2.5.

It remains to prove the displayed formula. We identify $L^{2}(G ; \mathrm{H})$ with $L^{2}(G) \otimes \mathrm{H}$ and $L^{2}(G ; \mathrm{K})$ with $L^{2}(G) \otimes \mathrm{K}$, and let $\xi \in \mathrm{H}, \eta \in \mathrm{K}$ and $f, h \in L^{2}(G)$. Then

$$
\left\langle h \otimes \eta, \pi_{\alpha}(x)(f \otimes \xi)\right\rangle=\int\left\langle h(g) \eta,\left(\pi_{\alpha}(x)(f \otimes \xi)\right)(g)\right\rangle d g .
$$

By Theorem 2.5, the left-hand side of the previous identity is equal to

$$
\int f(g) \overline{h(g)}\left\langle\eta, \alpha_{g^{-1}}(x) \xi\right\rangle d g=\int\left\langle h(g) \eta, f(g) \alpha_{g^{-1}}(x) \xi\right\rangle d g .
$$

Then the displayed formula follows by density.

In the next lemma we show how implemented actions of $G$ on $\mathrm{W}^{*}$-TROs look like.

Lemma 2.7. Assume that $\mathrm{X}$ is a concrete $W^{*}$-TRO in $B(\mathrm{H} ; \mathrm{K})$, that $\sigma: G \rightarrow B(\mathrm{H}), \tau: G \rightarrow$ $B(\mathrm{~K})$ are so-continuous representations of $G$ and that for each $g \in G$ and $x \in \mathrm{X}$ the operator $\tau_{g} x \sigma_{g}^{*}$ belongs to $\mathrm{X}$. Then the map $\alpha: G \rightarrow \operatorname{Aut}(\mathbf{X})$ defined by

$$
\alpha_{g}(x)=\tau_{g} x \sigma_{g}^{*}, \quad g \in G, x \in \mathrm{X},
$$

is an action of $G$ on $\mathrm{X}$.

Proof. Straightforward checks: we first observe that $\alpha_{g}$ is indeed a normal TRO automorphism of $\mathrm{X}$ and then verify $\alpha$ is a homomorphism and that the continuity conditions are satisfied.

In fact all actions of groups on TROs can be put in this form, at the cost of extending of the TRO in question. This is analogous to the crossed product construction for the actions of groups on von Neumann subalgebras.

Lemma 2.8. Let $\alpha: G \rightarrow \operatorname{Aut}(\mathrm{X})$ be an action of a locally compact group $G$ on a $W^{*}-T R O$ $\mathrm{X}$. Assume that $\mathrm{X}$ is concretely represented as a $W^{*}$-sub-TRO of $B(\mathrm{H} ; \mathrm{K})$. Let $\pi:=\pi_{\alpha}$ : $\mathrm{X} \rightarrow B\left(L^{2}(G) \otimes \mathrm{H} ; L^{2}(G) \otimes \mathrm{K}\right)$ be the representation of $\mathrm{X}$ introduced Proposition 2.6 and let $\tau=\lambda \otimes I_{\mathrm{K}}, \sigma=\lambda \otimes I_{\mathrm{H}}$ denote the respective amplifications of the left regular representation of $G$. Then the space

$$
\mathrm{w}^{*}-\mathrm{cl} \operatorname{Lin}\left\{\left(\mathrm{VN}(G) \otimes I_{\mathrm{K}}\right) \pi(\mathrm{X})\right\}
$$


is equal to

$$
\begin{aligned}
\mathrm{w}^{*}-\mathrm{cl} \operatorname{Lin}\left\{\tau_{g} \pi(x): g \in G, x \in \mathrm{X}\right\} & =\mathrm{w}^{*}-\mathrm{cl} \operatorname{Lin}\left\{\tau_{g} \pi(x) \sigma_{g^{\prime}}: g, g^{\prime} \in G\right\} \\
& =\mathrm{w}^{*}-\mathrm{cl} \operatorname{Lin}\left\{\pi(x) \sigma_{g}: g \in G, x \in \mathrm{X}\right\}
\end{aligned}
$$

and is a $W^{*}$-TRO. Moreover, we have the following formula:

$$
\pi\left(\alpha_{g}(x)\right)=\tau_{g} \pi(x) \sigma_{g}^{*}, \quad g \in G, x \in \mathrm{X} .
$$

Proof. It suffices to prove the formula (2.5), the rest is based on easy checks.

For $x \in \mathrm{X}, \xi \in L^{2}(G ; \mathrm{H})$ and a.e. $g, h \in G$, we have

$$
\begin{aligned}
\left(\tau_{g} \pi(x) \sigma_{g}^{*} \xi\right)(h) & =\left(\pi(x) \sigma_{g^{-1}} \xi\right)\left(g^{-1} h\right)=\alpha_{h^{-1} g}(x)\left(\left(\sigma_{g^{-1}} \xi\right)\left(g^{-1} h\right)\right) \\
& =\alpha_{h^{-1}}\left(\alpha_{g}(x)\right)(\xi(h))=\pi\left(\alpha_{g}(x) \xi\right)(h)
\end{aligned}
$$

as claimed.

Definition 2.9. Let $\alpha: G \rightarrow \operatorname{Aut}(\mathrm{X})$ be an action of a locally compact group $G$ on a $\mathrm{W}^{*}$ TRO X. The $\mathrm{W}^{*}$-TRO described by formula (2.4) above is called the crossed product of $\mathrm{X}$ by $\alpha$ and is denoted $G \ltimes_{\alpha}$ X.

Proposition 2.10. Let $\alpha: G \rightarrow \operatorname{Aut}(\mathrm{X})$ be an action of a locally compact group $G$ on a $W^{*}$-TRO X and let $\beta: G \rightarrow \operatorname{Aut}\left(\mathrm{R}_{\mathrm{X}}\right)$ be an action of $G$ on $\mathrm{R}_{\mathrm{X}}$ introduced in Theorem [2.3. Then the crossed product $G \ltimes_{\alpha} \mathrm{X}$ is the corner in the crossed product $G \ltimes_{\beta} \mathrm{R}_{\mathrm{X}}$ : if we start from $\mathrm{X}$ represented non-degenerately in $B(\mathrm{H} ; \mathrm{K})$, we obtain

$$
\left(I_{L^{2}(G)} \otimes P_{\mathrm{K}}\right)\left(G \ltimes_{\beta} \mathrm{R}_{\mathrm{X}}\right)\left(I_{L^{2}(G)} \otimes P_{\mathrm{H}}\right)=G \ltimes_{\alpha} \mathrm{X} .
$$

Proof. An immediate consequence of the fact that the space

$$
\left(I_{L^{2}(G)} \otimes P_{\mathrm{K}}\right)\left(\left(\mathrm{VN}(G) \otimes I_{\mathrm{K} \oplus \mathrm{H}}\right) \pi_{\beta}\left(\mathrm{R}_{\mathrm{X}}\right)\right)\left(I_{L^{2}(G)} \otimes P_{\mathrm{H}}\right)
$$

coincides with $\left(\mathrm{VN}(G) \otimes I_{\mathrm{K}}\right) \pi_{\alpha}(\mathrm{X})$, established in the proof of Theorem 2.5 and the weak* density of respective spaces.

The following corollary is now easy to observe, once again using the von Neumann algebra result and referring to the properties of Hamana extensions.

Corollary 2.11. The crossed product $G \ltimes_{\alpha} \mathrm{X}$ does not depend on the choice of the original faithful non-degenerate representation of $\mathrm{X}$.

The final result in this section explains the connection between the definition of the crossed product introduced above and that of Hamana in $\mathrm{Ha}_{2}$. Before we formulate it we need to introduce another action: if $\alpha: G \rightarrow \operatorname{Aut}(\mathrm{X})$ is an action, then $\operatorname{Ad}_{\rho} \otimes \alpha$ is an action of $G$ on the $\mathrm{W}^{*}$-TRO $B\left(L^{2}(G)\right) \bar{\otimes} \mathrm{X}$ given by the formula

$$
\left(\operatorname{Ad}_{\rho} \otimes \alpha\right)_{g}(z)=\left(\operatorname{Ad}_{\rho_{g}} \otimes \alpha_{g}\right)(z), \quad z \in B\left(L^{2}(G)\right) \bar{\otimes} \mathbf{X},
$$

where $\rho: G \rightarrow B\left(L^{2}(G)\right)$ is the right regular representation and we take the convention that $\operatorname{Ad}_{\rho_{g}}(z)=\rho_{g} z \rho_{g}^{*}$ for $z \in B\left(L^{2}(G)\right)$. Note for further use the following fact: if we write $\delta:=\operatorname{Ad}_{\rho} \otimes \alpha$, then the corresponding map $\pi_{\delta}: B\left(L^{2}(G)\right) \bar{\otimes} \mathbf{X} \rightarrow L^{\infty}(G) \bar{\otimes} B\left(L^{2}(G)\right) \bar{\otimes} \mathbf{X}$ is given explicitly by the formula:

$$
\pi_{\delta}(z)=\chi_{12}\left(V_{12}^{*}\left(\operatorname{id}_{B\left(L^{2}(G)\right)} \otimes \pi_{\alpha}\right)(z) V_{12}\right), \quad z \in B\left(L^{2}(G)\right) \bar{\otimes} \mathbf{X}
$$

where $V \in B\left(L^{2}(G) \otimes L^{2}(G)\right)$ is the right multiplicative unitary of $G$ (see Subsection 3 ) and $\chi_{12}$ flips the first two legs of the tensor product. 
Proposition 2.12. If $\alpha: G \rightarrow \operatorname{Aut}(\mathrm{X})$ is an action of $G$ on a $W^{*}-T R O \mathrm{X}$, then the following equality holds:

$$
G \ltimes_{\alpha} \mathrm{X}=\operatorname{Fix}\left(\operatorname{Ad}_{\rho} \otimes \alpha\right) .
$$

Proof. Assume that $\mathrm{X}$ is non-degenerately represented in $B(\mathrm{H} ; \mathrm{K})$ and consider the extension of $\alpha$ to an action $\beta$ of $G$ on the von Neumann algebra $\mathrm{R}_{\mathrm{X}}$ given by Lemma 2.3. The uniqueness of Hamana extensions shows that the action $\operatorname{Ad}_{\rho} \otimes \beta$ of $G$ on $B\left(L^{2}(G)\right) \bar{\otimes} \mathrm{R}_{\mathrm{X}}$ is the canonical extension of $\operatorname{Ad}_{\rho} \otimes \alpha$, the action of $G$ on $B\left(L^{2}(G)\right) \bar{\otimes}$. The left version of Corollary 19.13 in Str], attributed there to M. Takesaki and T. Digernes, shows that $G \ltimes_{\beta} \operatorname{RX}_{\mathbf{X}}=\operatorname{Fix}\left(\operatorname{Ad}_{\rho} \otimes \beta\right)$. In view of Proposition 2.10 it remains to show that

$$
\operatorname{Fix}\left(\operatorname{Ad}_{\rho} \otimes \alpha\right)=\left(I_{L^{2}(G)} \otimes P_{\mathrm{K}}\right) \operatorname{Fix}\left(\operatorname{Ad}_{\rho} \otimes \beta\right)\left(I_{L^{2}(G)} \otimes P_{\mathrm{H}}\right) .
$$

This however follows from Corollary 2.4 in view of the comments above.

Remark 2.13. In $\mathrm{Ha}_{2}$ Hamana defines the crossed product for an action of a group on an operator space directly via the fixed point formula of the type above. Note however that his definition does not coincide explicitly with ours, as he follows the approach of [NaT], where everything is formulated in terms of the right invariant Haar measure of $G$ (so that the crossed product contains the amplification of the right group von Neumann algebra).

It should be clear from the above discussions that it is also possible to develop the TRO crossed product construction in the $\mathrm{C}^{*}$-setting, we will however not need it in the sequel.

\section{Actions of locally compact quantum groups on $\mathrm{W}^{*}$-TROs and Resulting CROSSED PRODUCTS}

In this section we discuss actions of locally compact quantum groups on $\mathrm{W}^{*}$-TROs and define associated crossed products.

We follow the von Neumann algebraic approach to locally compact quantum groups due to Kustermans and Vaes $\mathrm{KuV}$, see also $\mathrm{KNR}_{1}$ and $\mathrm{KNR}_{2}$ for more background. A locally compact quantum group $\mathbb{G}$, effectively a virtual object, is studied via the von Neumann algebra $L^{\infty}(\mathbb{G})$, playing the role of the algebra of essentially bounded measurable functions on $\mathbb{G}$, equipped with a coproduct $\Delta: L^{\infty}(\mathbb{G}) \rightarrow L^{\infty}(\mathbb{G}) \bar{\otimes} L^{\infty}(\mathbb{G})$, which is a unital normal coassociative *-homomorphism. A locally compact quantum group $\mathbb{G}$ is by definition assumed to admit a left Haar weight $\phi$ and a right Haar weight $\psi$ - these are faithful, normal semifinite weights on $L^{\infty}(\mathbb{G})$ satisfying suitable invariance conditions. The GNS representation space for the left Haar weight will be denoted by $L^{2}(\mathbb{G})$. All the information about $\mathbb{G}$ is contained in the right multiplicative unitary $V \in B\left(L^{2}(\mathbb{G}) \otimes L^{2}(\mathbb{G})\right)$; it is a unitary operator such that we have

$$
\Delta(x)=V\left(x \otimes I_{L^{2}(\mathbb{G})}\right) V^{*}, \quad x \in L^{\infty}(\mathbb{G}) .
$$

This fact enables us to define a natural extension of the coproduct, the map $\widetilde{\Delta}: B\left(L^{2}(\mathbb{G})\right) \rightarrow$ $B\left(L^{2}(\mathbb{G}) \otimes L^{2}(\mathbb{G})\right)$ given by the same formula:

$$
\widetilde{\Delta}(y)=V\left(y \otimes I_{L^{2}(\mathbb{G})}\right) V^{*}, \quad y \in B\left(L^{2}(\mathbb{G})\right) .
$$

In fact, $\widetilde{\Delta}$ takes values in $B\left(L^{2}(\mathbb{G})\right) \bar{\otimes} L^{\infty}(\mathbb{G})$ as $V \in L^{\infty}(\widehat{\mathbb{G}})^{\prime} \bar{\otimes} L^{\infty}(\mathbb{G})$, where $\widehat{\mathbb{G}}$ is the $d u a l$ locally compact quantum group of $\mathbb{G}$ (the algebra $L^{\infty}(\widehat{\mathbb{G}})$ acts naturally on $L^{2}(\mathbb{G})$ ). If $\mathbb{G}=G$ happens to be a locally compact group, then $L^{\infty}(\widehat{\mathbb{G}})=\mathrm{VN}(G)$. Finally note that by analogy with the classical situation we denote the predual of $L^{\infty}(\mathbb{G})$ by $L^{1}(\mathbb{G})$. 
Recall the standard definition of an action of $\mathbb{G}$ on a von Neumann algebra M. A (continuous, left) action of $\mathbb{G}$ on $\mathrm{M}$ is an injective, normal, unital ${ }^{*}$-homomorphism $\beta: \mathrm{M} \rightarrow$ $L^{\infty}(\mathbb{G}) \bar{\otimes} \mathrm{M}$ satisfying the action equation

$$
\left(\operatorname{id}_{L^{\infty}(\mathbb{G})} \otimes \beta\right) \circ \beta=\left(\Delta_{\mathbb{G}} \otimes \operatorname{id}_{M}\right) \circ \beta .
$$

Replacing a von Neumann algebra with a $\mathrm{W}^{*}$-TRO yields no extra complications.

Definition 3.1. Let $X$ be a $W^{*}-T R O$ and let $\mathbb{G}$ be a locally compact quantum group. An action of $\mathbb{G}$ on $\mathbf{X}$ is an injective, normal, non-degenerate TRO morphism $\alpha: \mathbf{X} \rightarrow L^{\infty}(\mathbb{G}) \bar{\otimes} \mathbf{X}$ such that

$$
\left(\operatorname{id}_{L^{\infty}(\mathbb{G})} \otimes \alpha\right) \circ \alpha=\left(\Delta_{\mathbb{G}} \otimes \operatorname{id}_{M}\right) \circ \alpha .
$$

Theorem 2.5 implies that if $\mathbb{G}=G$ happens to be a classical locally compact group, the definition above agrees with Definition 2.1.

Proposition 3.2. Let $\mathrm{X}$ be $a W^{*}-T R O$ and let $\mathbb{G}$ be a locally compact quantum group acting on $\mathbf{X}$ via $\alpha: \mathrm{X} \rightarrow L^{\infty}(\mathbb{G}) \bar{\otimes} \mathbf{X}$. Then the extension provided by Proposition 1.2 defines an action $\beta=\mathrm{R}_{\mathrm{X}} \rightarrow L^{\infty}(\mathbb{G}) \bar{\otimes} \mathrm{R}_{\mathrm{X}}$ of $\mathbb{G}$ on $\mathrm{R}_{\mathrm{X}}$.

Proof. Similar to the proof of Theorem 2.5. effectively we use the fact that if we denote the $\mathrm{W}^{*}$-TRO $L^{\infty}(\mathbb{G}) \bar{\otimes} \mathrm{X}$ by $\mathrm{Y}$, then we have $\mathrm{R}_{\mathrm{Y}} \cong L^{\infty}(\mathbb{G}) \bar{\otimes} \mathrm{R}_{\mathrm{X}}$.

If $\beta: \mathrm{M} \rightarrow L^{\infty}(\mathbb{G}) \bar{\otimes} \mathrm{M}$ is an action of $\mathbb{G}$ on a von Neumann algebra $\mathrm{M}$, the crossed product $\mathbb{G} \ltimes_{\beta} \mathrm{M}$ is defined as the von Neumann algebra $\left(\left(L^{\infty}(\widehat{\mathbb{G}}) \otimes I_{\mathrm{M}}\right) \beta(\mathrm{M})\right)^{\prime \prime}$. Equivalently,

$$
\mathbb{G} \ltimes_{\beta} \mathrm{M}=\mathrm{w}^{*}-\mathrm{cl} \operatorname{Lin}\left\{\left(y \otimes I_{\mathrm{M}}\right) \beta(m): y \in L^{\infty}(\widehat{\mathbb{G}}), m \in \mathrm{M}\right\} .
$$

The last equality amounts to the fact that the weak* closure of $\left(L^{\infty}(\widehat{\mathbb{G}}) \otimes I_{\mathbf{M}}\right) \beta(\mathrm{M})$ is a *subspace of $B\left(L^{2}(\mathbb{G})\right) \bar{\otimes}$. This is a well-known fact, formulated for example in Proposition 2.3 of [KaS]: it can be shown using a simpler version of the $\mathrm{C}^{*}$-algebraic calculations in [SkZ] after Definition 2.4 .

Definition 3.3. Let $\mathrm{X}$ be a $\mathrm{W}^{*}$-TRO non-degenerately represented in $B(\mathrm{H} ; \mathrm{K})$ and let $\mathbb{G}$ be a locally compact quantum group acting on $\mathbf{X}$ via $\alpha: \mathbf{X} \rightarrow L^{\infty}(\mathbb{G}) \bar{\otimes}$. The $W^{*}$-TRO crossed product $\mathbb{G} \ltimes_{\alpha} \mathbf{X}$ is defined as the weak* closure of the linear span of $\left(L^{\infty}(\widehat{\mathbb{G}}) \otimes I_{\mathrm{K}}\right) \alpha(\mathrm{X})$.

We will soon note that again the crossed product has several other descriptions, but we first need to record the quantum version of Proposition 2.10.

Proposition 3.4. Let $\mathrm{X}$ be a $W^{*}-T R O$ non-degenerately represented in $B(\mathrm{H} ; \mathrm{K})$ and let $\mathbb{G}$ be a locally compact quantum group acting on $\mathbf{X}$ via $\alpha: \mathbf{X} \rightarrow L^{\infty}(\mathbb{G}) \bar{\otimes} \mathbf{X}$. Let $\beta: \mathrm{R}_{\mathbf{X}} \rightarrow$ $L^{\infty}(\mathbb{G}) \bar{\otimes} \mathrm{R}_{\mathrm{X}}$ be the action of $\mathbb{G}$ on $\mathrm{R}_{\mathrm{X}}$ provided by Proposition 3.2. Then

$$
\left(I_{L^{2}(G)} \otimes P_{\mathrm{K}}\right)\left(\mathbb{G} \ltimes_{\beta} \mathrm{R}_{\mathrm{X}}\right)\left(I_{L^{2}(G)} \otimes P_{\mathrm{H}}\right)=\mathbb{G} \ltimes_{\alpha} \mathrm{X} .
$$

Proof. Follows exactly as in the case of Proposition 2.10.

Corollary 3.5. Under the assumptions of Definition 3.3, we have the following equalities:

$$
\begin{aligned}
\mathbb{G} \ltimes_{\alpha} \mathrm{X} & =\mathrm{w}^{*}-\mathrm{cl} \operatorname{Lin}\left\{\left(L^{\infty}(\widehat{\mathbb{G}}) \otimes I_{\mathrm{K}}\right) \alpha(\mathrm{X})\left(L^{\infty}(\widehat{\mathbb{G}}) \otimes I_{\mathrm{H}}\right)\right\} \\
& =\mathrm{w}^{*}-\mathrm{cl} \operatorname{Lin}\left\{\alpha(\mathbf{X})\left(L^{\infty}(\widehat{\mathbb{G}}) \otimes I_{\mathrm{H}}\right)\right\} .
\end{aligned}
$$

Moreover, $\mathbb{G} \ltimes_{\alpha} \mathrm{X}$ is the $W^{*}$-TRO generated in $B\left(L^{2}(\mathbb{G}) \otimes \mathrm{H} ; L^{2}(\mathbb{G}) \otimes \mathrm{K}\right)$ by the set $\left(L^{\infty}(\widehat{\mathbb{G}}) \otimes\right.$ $\left.I_{\mathrm{K}}\right) \alpha(\mathrm{X})$. It does not depend (up to an isomorphism) on the initial choice of a faithful nondegenerate representation of $\mathrm{X}$. 
Proof. Follows immediately from the analogous facts for the von Neumann crossed products and Proposition 3.4 .

If $\alpha: \mathrm{X} \rightarrow L^{\infty}(\mathbb{G}) \bar{\otimes} \mathbf{X}$ is an action of $\mathbb{G}$ on a $\mathrm{W}^{*}$-TRO $\mathbf{X}$, then the fixed point space of $\alpha$ is defined as

$$
\text { Fix } \alpha=\left\{x \in \mathbf{X}: \alpha(x)=I_{L^{2}(\mathbb{G})} \otimes x\right\} .
$$

Proposition 3.6. Assume that $\mathrm{X}$ is a $W^{*}$-TRO non-degenerately represented in some $B(\mathrm{H} ; \mathrm{K})$, $\alpha$ is an action of $\mathbb{G}$ on $\mathbf{X}$ and $\beta$ is an action of $\mathbb{G}$ on $\mathrm{R}_{\mathbf{X}}$ introduced in Proposition 3.2. Then Fix $\alpha=P_{\mathrm{K}}(\operatorname{Fix} \beta) P_{\mathrm{H}}$.

Proof. Follows as in Corollary 2.4.

Let $\alpha: \mathbf{X} \rightarrow L^{\infty}(\mathbb{G}) \bar{\otimes} \mathbf{X}$ be an action of $\mathbb{G}$ on $\mathbf{X}$ and consider the following map $\delta$ : $B\left(L^{2}(\mathbb{G})\right) \bar{\otimes} \mathbf{X} \rightarrow L^{\infty}(\mathbb{G}) \bar{\otimes} B\left(L^{2}(\mathbb{G})\right) \bar{\otimes} \mathbf{X}:$

$$
\delta(z)=\chi_{12}\left(V_{12}^{*}\left(\operatorname{id}_{B\left(L^{2}(G)\right)} \otimes \alpha\right)(z) V_{12}\right), \quad z \in B\left(L^{2}(\mathbb{G})\right) \bar{\otimes} \mathbf{X},
$$

where $V$ is the right multiplicative unitary of $\mathbb{G}$ (compare to the formula (2.7), remembering that for quantum groups we denote simply by $\alpha$ what used to be $\pi_{\alpha}$ ).

The following result is a quantum version of Proposition 2.12, this time following from the von Neumann algebraic result due to Enock [Eno, see also $\mathrm{KNR}_{2}$.

Theorem 3.7. Let $\mathrm{X}$ be a $W^{*}-T R O$ and let $\mathbb{G}$ be a locally compact quantum group acting on $\mathbf{X}$ via $\alpha: \mathbf{X} \rightarrow L^{\infty}(\mathbb{G}) \bar{\otimes} \mathbf{X}$. The map $\delta: B\left(L^{2}(\mathbb{G})\right) \bar{\otimes} \mathbf{X} \rightarrow L^{\infty}(\mathbb{G}) \bar{\otimes} B\left(L^{2}(\mathbb{G})\right) \bar{\otimes} \mathbf{X}$ defined by (3.2) is an action of $\mathbb{G}$ on the $W^{*}-T R O B\left(L^{2}(\mathbb{G})\right) \bar{\otimes}$. Moreover we have the following equality:

$$
\mathbb{G} \ltimes_{\alpha} \mathrm{X}=\operatorname{Fix} \delta .
$$

Proof. Let $\mathrm{X}$ be non-degenerately represented in $B(\mathrm{H} ; \mathrm{K})$ and let $\beta$ be the action of $\mathbb{G}$ on $\mathrm{R}_{\mathrm{X}}$ provided in Theorem 3.2 . Then Theorem 2.3 of $\mathrm{KNR}_{2}$, which is a simplified version of Theorem 11.6 of [Eno], says that the map $\gamma: B\left(L^{2}(\mathbb{G})\right) \bar{\otimes} \mathrm{R}_{\mathbf{X}} \rightarrow L^{\infty}(\mathbb{G}) \bar{\otimes} B\left(L^{2}(\mathbb{G})\right) \bar{\otimes} \mathrm{R}_{\mathbf{X}}$ :

$$
\gamma(t)=\chi_{12}\left(V_{12}^{*}\left(\operatorname{id}_{B\left(L^{2}(G)\right)} \otimes \beta\right)(t) V_{12}\right), \quad t \in B\left(L^{2}(\mathbb{G})\right) \bar{\otimes} \mathrm{R}_{\mathbf{X}},
$$

is an action of $\mathbb{G}$ on $R_{X}$ and

$$
\mathbb{G} \ltimes_{\beta} \mathrm{RX}_{\mathrm{X}}=\operatorname{Fix} \gamma .
$$

It is easy to verify that in fact for $z \in B\left(L^{2}(\mathbb{G})\right) \bar{\otimes} \mathrm{X} \subset B\left(L^{2}(\mathbb{G})\right) \bar{\otimes} \mathrm{R}_{\mathbf{X}}$ we have

$$
\delta(z)=\left(I_{L^{2}(\mathbb{G})} \otimes I_{L^{2}(\mathbb{G})} \otimes P_{\mathrm{K}}\right) \gamma(z)\left(I_{L^{2}(\mathbb{G})} \otimes I_{L^{2}(\mathbb{G})} \otimes P_{\mathrm{H}}\right) .
$$

This implies, via Corollary 1.3, that $\delta$ is a normal TRO morphism, whose Hamana extension is $\gamma$. An explicit computation and another application of Proposition 1.2 show that $\delta$ is in fact an action of $\mathbb{G}$ on the $\mathrm{W}^{*}$-TRO $B\left(L^{2}(\mathbb{G})\right) \bar{\otimes}$, with $\gamma$ clearly being the extension of $\delta$ provided by Proposition [3.2. Then formula (3.4) and Propositions 3.4 and 3.6 end the proof.

We finish this section by discussing certain connections between the TROs arising as fixed point spaces of completely contractive maps, studied in Section 1, and (quantum) group actions. 
Proposition 3.8. Let $\mathrm{M}$ be a von Neumann algebra, and let $\beta: \mathrm{M} \rightarrow L^{\infty}(\mathbb{G}) \bar{\otimes} \mathrm{M}$ be an action of a locally compact quantum group on $\mathrm{M}$. Let $\widetilde{\mathrm{X}}$ be a weak ${ }^{*}$-closed subspace of $\mathrm{M}$, and let $P: \mathrm{M} \rightarrow \mathrm{M}$ be a completely contractive idempotent map such that $P(\mathrm{M})=\widetilde{\mathrm{X}}$ and

$$
\beta \circ P=\left(\operatorname{id}_{L^{\infty}(\mathbb{G})} \otimes_{F} P\right) \circ \beta .
$$

Then the formula (see Proposition [1.5)

$$
\alpha=\left(\operatorname{id}_{L^{\infty}(\mathbb{G})} \bar{\otimes} \iota\right) \circ \beta \circ \iota^{-1}
$$

defines a normal, injective TRO morphism $\alpha: \mathbf{X} \rightarrow L^{\infty}(\mathbb{G}) \bar{\otimes} \mathbf{X}$ satisfying the action equation

$$
\left(\operatorname{id}_{L^{\infty}(\mathbb{G})} \otimes \alpha\right) \circ \alpha=\left(\Delta_{\mathbb{G}} \otimes \operatorname{id}_{M}\right) \circ \alpha .
$$

Proof. For $x, y, z \in \widetilde{\mathrm{X}}$, we have

$$
\begin{aligned}
\alpha\left(\{\iota(x), \iota(y), \iota(z)\}_{\mathbf{X}}\right) & =\left(\operatorname{id}_{L^{\infty}(\mathbb{G})} \bar{\otimes} \iota\right) \circ \beta\left(P\left(x y^{*} z\right)\right) \\
& =\left(\operatorname{id}_{L^{\infty}(\mathbb{G})} \bar{\otimes} \iota\right) \circ\left(\operatorname{id}_{L^{\infty}(\mathbb{G})} \otimes_{F} P\right)\left(\beta(x) \beta(y)^{*} \beta(z)\right) \\
& =\{\alpha(\iota(x)), \alpha(\iota(y)), \alpha(\iota(z))\}_{L^{\infty}(\mathbb{G}) \bar{\otimes} \mathbf{x}}
\end{aligned}
$$

Hence $\alpha$ is a TRO morphism, and it is normal because $\iota$ is a weak ${ }^{*}$-homeomorphism. It is also easy to check that $\alpha$ is injective and satisfies the coassociativity condition, using the corresponding properties of $\beta$.

Remark 3.9. We do not know whether the map constructed above is an action of $\mathbb{G}$ on $X$, as it is not clear whether it is non-degenerate. Let us sketch a natural approach to proving non-degeneracy, so that it is clear where it breaks down. Using the notations of the last proposition we should show that the weak*-closed linear span of elements of the form

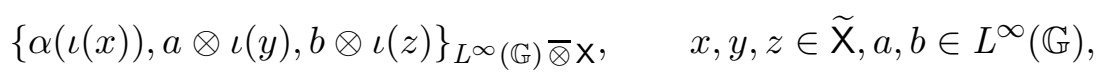

is equal to $L^{\infty}(\mathbb{G}) \bar{\otimes} \mathrm{X}$. Writing $x=P(m)$ for $m \in \mathrm{M}$, we have

$$
\begin{aligned}
\{\alpha(\iota(x)), a \otimes \iota(y), b \otimes \iota(z)\}_{L^{\infty}(\mathbb{G}) \bar{\otimes}} & =\left\{\left(\operatorname{id}_{L^{\infty}(\mathbb{G})} \otimes \iota P\right) \beta(m), a \otimes \iota(y), b \otimes \iota(z)\right\}_{L^{\infty}(\mathbb{G}) \bar{X} \mathbf{x}} \\
& =\left(\operatorname{id}_{L^{\infty}(\mathbb{G})} \otimes \iota P\right)\left(\beta(m)\left(a^{*} b \otimes y^{*} z\right)\right)
\end{aligned}
$$

(where we used Youngson's identity $P\left(P\left(m_{1}\right) P\left(m_{2}\right)^{*} P\left(m_{3}\right)\right)=P\left(m_{1} P\left(m_{2}\right)^{*} P\left(m_{3}\right)\right)$ ). In other words, it suffices to show that $(\mathrm{id} \otimes P)\left(\beta(\mathrm{M})\left(L^{\infty}(\mathbb{G}) \bar{\otimes} \widetilde{\mathrm{X}} * \widetilde{\mathrm{X}}\right)\right)$ is linearly weak*-dense in $L^{\infty}(\mathbb{G}) \bar{\otimes} \widetilde{\mathrm{X}}$. Now we know on one hand via Proposition 2.9 of $\left[\mathrm{KaS}\right.$ that $\beta(\mathrm{M})\left(L^{\infty}(\mathbb{G}) \otimes 1\right)$ is linearly weak*-dense in $L^{\infty}(\mathbb{G}) \bar{\otimes} \mathrm{M}$ and on the other hand that $P\left(\mathrm{MX} \widetilde{X}^{*} \widetilde{X}\right)$ is weak*-dense in $\widetilde{\mathrm{X}}$ (essentially because $\mathrm{X}$ is a $\mathrm{W}^{*}$-TRO). Combining these two facts brings us close to completing the proof, but $P$ is not assumed to be normal (and cannot be for applications). Note that in the positive case (by which we mean the case where $P$ is a completely positive projection and $\mathrm{X}$ is a von Neumann algebra) the argument goes through simply by choosing $m=1$.

The problem disappears in the case $\mathbb{G}$ is a classical group, as then we can rather use the pointwise picture of the actions.

Theorem 3.10. Let $\mathrm{M}$ be a von Neumann algebra, and let $\beta: G \rightarrow \operatorname{Aut}(\mathrm{M})$ be an action of a locally compact group $G$ on $\mathrm{M}$. Let $\widetilde{\mathrm{X}}$ be a weak*-closed subspace of $\mathrm{M}$, and let $P: \mathrm{M} \rightarrow \mathrm{M}$ be a completely contractive idempotent map such that $P(\mathrm{M})=\widetilde{\mathrm{X}}$ and

$$
\beta_{g} \circ P=P \circ \beta_{g}, \quad g \in G .
$$


Then the formula (see Proposition [1.5)

$$
\alpha_{g}=\iota \circ \beta_{g} \circ \iota^{-1}, \quad g \in G,
$$

defines an action $\alpha$ of $G$ on the $W^{*}-T R O X$.

Proof. The fact that each $\alpha_{g}(g \in G)$ is a normal TRO morphism follows as in the last proposition; as we have for $g, h \in G$

$$
\alpha_{g} \circ \alpha_{h}=\iota \circ \beta_{g} \circ \iota^{-1} \circ \iota \circ \beta_{h} \circ \iota^{-1}=\iota \circ \beta_{g} \circ \beta_{h} \circ \iota^{-1}=\iota \circ \beta_{g h} \circ \iota^{-1}=\alpha_{g h}
$$

and $\alpha_{e}=\mathrm{idx}$, each $\alpha_{g}$ is in fact an automorphism, and $\alpha: G \rightarrow \operatorname{Aut}(\mathrm{X})$ is a homomorphism. Finally the continuity condition follows from that for $\beta$ : if $\left(g_{i}\right)_{i \in \mathcal{I}}$ is a net of elements of $G$ converging to $g \in G$ and $x \in \mathrm{X}$, then, as $\iota$ is a homeomorphism with respect to weak *-topologies, we have

$$
\begin{aligned}
\mathrm{w}^{*}-\lim _{i \in \mathcal{I}} \alpha^{x}\left(g_{i}\right) & =\mathrm{w}^{*}-\lim _{i \in \mathcal{I}} \alpha_{g_{i}}(x)=\mathrm{w}^{*}-\lim _{i \in \mathcal{I}} \iota\left(\beta_{g_{i}}\left(\iota^{-1}(x)\right)\right)=\iota\left(\mathrm{w}^{*}-\lim _{i \in \mathcal{I}} \beta_{g_{i}}\left(\iota^{-1}(x)\right)\right) \\
& =\iota\left(\beta_{g}\left(\iota^{-1}(x)\right)\right)=\alpha^{x}(g) .
\end{aligned}
$$

Note that although the projection $P$ features in one of the conditions both in Proposition 3.8 and Theorem 3.10, the actual maps constructed there depend only on its image.

\section{Poisson boundaries associated with contractive functionals in $C_{0}^{u}(\mathbb{G})^{*}$}

Let $\mathbb{G}$ be a locally compact quantum group, let $C_{0}^{u}(\mathbb{G})$ be the universal $\mathrm{C}^{*}$-algebra associated with $\mathbb{G}$, and let $\Delta_{u}$ be the coproduct on $C_{0}^{u}(\mathbb{G})$ (see $[\mathrm{Kus}$ ). The Banach space dual of $C_{0}^{u}(\mathbb{G})$ will be denoted $M^{u}(\mathbb{G})$ and called the measure algebra of $\mathbb{G}$. It is a Banach algebra with the product defined by

$$
\mu \star \nu:=(\mu \otimes \nu) \circ \Delta_{u}, \quad \mu, \nu \in M^{u}(\mathbb{G}) .
$$

Given $\mu \in M^{u}(\mathbb{G})$ the associated right convolution operator $R_{\mu}: L^{\infty}(\mathbb{G}) \rightarrow L^{\infty}(\mathbb{G})$ is defined by the formula

$$
\left\langle R_{\mu}(x), \omega\right\rangle=\langle\omega \star \mu, x\rangle, \quad x \in L^{\infty}(\mathbb{G}), \omega \in L^{1}(\mathbb{G}) .
$$

This is well-defined as $L^{1}(\mathbb{G})$ is an ideal in $M^{u}(\mathbb{G})$. Moreover, $R_{\mu}$ is normal.

We are ready to apply the results of the earlier sections to the construction of extended Poisson boundaries for contractive (not-necessarily positive) quantum measures. We say that $\mu \in M^{u}(\mathbb{G})$ is contractive if $\|\mu\| \leq 1$.

Theorem 4.1. Let $\mu \in M^{u}(\mathbb{G})$ be contractive. Then the fixed point space Fix $R_{\mu}:=\{x \in$ $\left.L^{\infty}(\mathbb{G}): R_{\mu}(x)=x\right\}$ has a unique (up to a weak*-continuous complete isometry) structure of a $W^{*}-T R O$, which we will denote $\mathbf{X}_{\mu}$.

Proof. This is an immediate consequence of Theorem 1.6, as $R_{\mu}: L^{\infty}(\mathbb{G}) \rightarrow L^{\infty}(\mathbb{G})$ is a complete contraction.

We will call the space Fix $R_{\mu}$ with the $\mathrm{W}^{*}$-TRO structure induced via Theorem 4.1 an extended Poisson boundary associated to $\mu$.

Lemma 4.2. Let $\mu \in M^{u}(\mathbb{G})$ be contractive. The extended Poisson boundary Fix $R_{\mu}$ is a unital subspace of $L^{\infty}(\mathbb{G})$ iff $\mu$ is a state. 
Proof. Follows from the equivalence $R_{\mu}\left(I_{L^{\infty}(\mathbb{G})}\right)=I_{L^{\infty}(\mathbb{G})}$ iff $\mu\left(I_{M C_{0}^{u}(\mathbb{G})}\right)=1$ iff $\mu$ is a state (here $M C_{0}^{u}(\mathbb{G})$ denotes the multiplier algebra of $C_{0}^{u}(\mathbb{G})$ ).

Corollary 4.3. A locally compact quantum group $\mathbb{G}$ is amenable if and only if there exists a contractive $\mu \in M^{u}(\mathbb{G})$ such that Fix $R_{\mu}=\mathbb{C} I_{L^{\infty}(\mathbb{G})}$.

Proof. Follows from the last lemma and Theorem 4.2 of $\left[\mathrm{KNR}_{1}\right]$.

Given a contractive $\mu \in M^{u}(\mathbb{G})$ we can also consider an associated convolution operator $\Theta_{\mu}$ acting on $B\left(L^{2}(\mathbb{G})\right.$ ), defined in $\left[\mathrm{JNR}\right.$. (see also $\mathrm{KNR}_{2}$ ): it is a unique normal completely bounded map such that

$$
\widetilde{\Delta} \circ \Theta_{\mu}=\left(\operatorname{id}_{B\left(L^{2}(\mathbb{G})\right)} \otimes R_{\mu}\right) \circ \widetilde{\Delta} .
$$

Theorem 4.4. Let $\mu \in M^{u}(\mathbb{G})$ be contractive. Then the fixed point space Fix $\Theta_{\mu}:=\{x \in$ $\left.B\left(L^{2}(\mathbb{G})\right): \Theta_{\mu}(x)=x\right\}$ has a unique (up to a weak*-continuous complete isometry) structure of a $W^{*}-T R O$, which we will denote $\mathrm{Y}_{\mu}$.

Proof. This is an immediate consequence of Theorem 1.6, as $\Theta_{\mu}: B\left(L^{2}(\mathbb{G})\right) \rightarrow B\left(L^{2}(\mathbb{G})\right)$ is a normal complete contraction.

Let $\mu \in M^{u}(\mathbb{G})$ be contractive. Fix a free ultrafilter $\beta$ and use it as in Theorem 1.6 to construct completely contractive projections $P$ from $L^{\infty}(\mathbb{G})$ onto Fix $R_{\mu}$ and $P_{\Theta}$ from $B\left(L^{2}(\mathbb{G})\right)$ onto Fix $\Theta_{\mu}$. It is then not difficult to see that due to (4.1) we have also

$$
\widetilde{\Delta} \circ P_{\Theta}=\left(\operatorname{id}_{B\left(L^{2}(\mathbb{G})\right)} \otimes_{F} P\right) \circ \widetilde{\Delta} .
$$

Proposition 4.5. Let $\mathbb{G}$ and $\mu$ be as above and let $\iota: \operatorname{Fix} R_{\mu} \rightarrow \mathrm{X}_{\mu}, \kappa:$ Fix $\Theta_{\mu} \rightarrow \mathrm{Y}_{\mu}$ denote respective weak $k^{*}$ homeomorphisms. Then the formula

$$
\gamma=\left(\operatorname{id}_{B\left(L^{2}(\mathbb{G})\right)} \otimes \iota\right) \circ \widetilde{\Delta} \circ \kappa^{-1}
$$

defines an injective normal TRO morphism $\gamma: \mathrm{Y}_{\mu} \rightarrow B\left(L^{2}(\mathbb{G})\right) \bar{\otimes} \mathrm{X}_{\mu}$.

Proof. This is proved similarly as Proposition 3.8, using the intertwining relation (4.2).

In the case where $G$ is a classical group and $\mu \in M(G)$ is contractive, we can in fact identify the image of the map $\gamma$. First of all we can show via Theorem 3.10 that there is a natural action of $G$ on the $\mathrm{W}^{*}$-TRO arising from Fix $R_{\mu}$.

Lemma 4.6. Let $G$ be a locally compact group and let $\mu \in M(G)$ be contractive. Then there is a natural action $\alpha$ of $G$ on the $W^{*}$-TRO $\mathrm{X}_{\mu}$, given essentially by the left multiplication.

Proof. Consider the action $\beta$ of $G$ on $L^{\infty}(G)$ given by the formula

$$
\left(\beta_{g}(f)\right)(h)=f\left(g^{-1} h\right), \quad f \in L^{\infty}(G), g, h \in G .
$$

It is then easy to check that we have $\beta_{g} \circ R_{\mu}=R_{\mu} \circ \beta_{g}$, and so also $\beta_{g} \circ P=P \circ \beta_{g}$, where $P$ is a completely contractive projection given by the limit (along some ultrafilter) of iterates of $R_{\mu}$. Theorem 3.10 ends the proof.

We are now ready to establish the connection between the $\mathrm{W}^{*}-$ TROs $\mathrm{X}_{\mu}$ and $\mathrm{Y}_{\mu}$.

Theorem 4.7. Let $G$ be a locally compact group and let $\mu \in M(G)$ be contractive. Let $\alpha$ be the action of $G$ on the $W^{*}-T R O \mathrm{X}_{\mu}$ introduced in Lemma 4.6. We then have a natural isomorphism

$$
\mathrm{Y}_{\mu} \cong G \ltimes_{\alpha} \mathrm{X}_{\mu}
$$

given by the map $\gamma$ introduced in Proposition 4.5 . 
Proof. We begin by showing that $\gamma\left(\mathrm{Y}_{\mu}\right)$ is contained in $G \ltimes_{\alpha} \mathrm{X}_{\mu}$. By Proposition 2.12 it suffices to show that for each $x \in \widetilde{\mathrm{Y}}_{\mu}$ and $g \in G$ we have $\left(\operatorname{Ad}_{\rho} \otimes \alpha\right)_{g}(\gamma(\kappa(x)))=\gamma(\kappa(x))$. Recall that by the definition of the action constructed in Lemma 4.6 we have $\alpha_{g} \circ \iota=\iota \circ \beta_{g}$. Recall also that if we view $L^{\infty}(G)$ as a subalgebra of $B\left(L^{2}(G)\right)$, then the map $\beta_{g}$ is equal to $\left(\operatorname{Ad}_{\lambda}\right)_{g}$, where $\lambda$ denotes again the left regular representation. This means that

$$
\left(\left(\operatorname{Ad}_{\rho}\right)_{g} \otimes \alpha_{g}\right)((\mathrm{id} \otimes \iota) \circ \widetilde{\Delta}(x))=(\mathrm{id} \otimes \iota)\left(\left(\rho_{g} \otimes \lambda_{g}\right) V(x \otimes 1) V^{*}\left(\rho_{g}^{*} \otimes \lambda_{g}^{*}\right)\right)
$$

Recall that the right multiplicative unitary for $G$ is given by the formula

$$
(V f)(g, h)=\delta(h)^{1 / 2} f(g h, h), \quad f \in L^{2}(G), g, h \in G,
$$

where $\delta$ is the modular function of $G$. Then an explicit calculation shows that for any $y \in B\left(L^{2}(G)\right)$ we have

$$
\left(\rho_{g} \otimes \lambda_{g}\right) V(y \otimes 1) V^{*}\left(\rho_{g}^{*} \otimes \lambda_{g}^{*}\right)=V(y \otimes 1) V^{*} .
$$

Thus $\left(\operatorname{Ad}_{\rho} \otimes \alpha\right)_{g}(\gamma(\kappa(x)))$ does not in fact depend on $g$ and the first part of the theorem is proved.

As $\gamma$ is a normal TRO morphism, it has a weak*-closed image. Thus to show that $\gamma\left(\mathrm{Y}_{\mu}\right)$ contains $\mathrm{X}_{\mu} \rtimes_{\alpha} G$ it suffices (by Lemma 2.8 and Definition 2.9) to show that for every $g \in G$ and $x \in$ Fix $R_{\mu}$ we have $\left(\lambda_{g} \otimes I\right)\left(\pi_{\alpha}(\iota(x)) \in \gamma\left(\mathrm{Y}_{\mu}\right)\right.$ (note that the symbol $I$ above can be interpreted explicitly once we fix a concrete representation of $\left.\mathrm{X}_{\mu}\right)$. This is equivalent to proving that $\left(\mathrm{id} \otimes \iota^{-1}\right)\left(\left(\lambda_{g} \otimes I\right)\left(\pi_{\alpha}(\iota(x))\right) \in \widetilde{\Delta}\left(\operatorname{Fix} \Theta_{\mu}\right)\right.$. Consider the map (id $\left.\otimes \iota^{-1}\right)$ : $B\left(L^{2}(G)\right) \bar{\otimes} \mathrm{X}_{\mu} \rightarrow B\left(L^{2}(G)\right) \bar{\otimes}$ Fix $R_{\mu}$. Note that both the domain and range spaces are in fact $B\left(L^{2}(G)\right)$ left modules in a natural way and moreover that (id $\otimes \iota^{-1}$ ) is a $B\left(L^{2}(G)\right)$-module map. Thus, recalling how the action $\alpha$ was constructed in Lemma 4.6 we can first verify that the integrated forms of actions $\alpha$ and $\beta$ satisfy the equality (id $\otimes \iota$ ) $\circ \pi_{\beta}=\pi_{\alpha} \circ \iota$ (remembering that $\iota$ is a homeomorphism for weak* topologies and using equality (2.10) and then see that $\left(\mathrm{id} \otimes \iota^{-1}\right)\left(\left(\lambda_{g} \otimes I\right)\left(\pi_{\alpha}(\iota(x))\right)=\left(\lambda_{g} \otimes I\right) \pi_{\beta}(x)\right.$. Now the integrated form of the action $\beta$ is nothing but the coproduct, so we need to show simply that $\left(\lambda_{g} \otimes I\right) \Delta(x) \in \widetilde{\Delta}\left(\operatorname{Fix} \Theta_{\mu}\right)$. To this end we consider $\lambda_{g} x \in B\left(L^{2}(G)\right)$. As $\Theta_{\mu}$ is a $\operatorname{VN}(G)$-module map which extends $R_{\mu}$ and $x \in$ Fix $R_{\mu}$, we have $\lambda_{g} x \in$ Fix $\Theta_{\mu}$. Then as the first leg of $V$ commutes with $\operatorname{VN}(G)$ we have $\widetilde{\Delta}\left(\lambda_{g} x\right)=\left(\lambda_{g} \otimes I\right) \widetilde{\Delta}(x)=\left(\lambda_{g} \otimes I\right) \Delta(x)$. This ends the proof.

Remark 4.8. The analogous result for $\mu$ being a state is shown in $\mathrm{KNR}_{1}$ for $G$ replaced by any locally compact quantum group. Here the stumbling block in extending the last theorem to the quantum setting is precisely the fact that we are not able to deduce in general that the map $\alpha$ constructed in Proposition 3.8 is non-degenerate (otherwise we could use Theorem 3.7 instead of Proposition 2.12).

\section{REFERENCES}

[BLM] D. Blecher and C. Le Merdy, Operator algebras and their modules an operator space approach, Oxford University Press, Oxford, 2004.

[ChL] C.H. Chu and A.T.-M. Lau, Harmonic functions on groups and Fourier algebras, Lecture Notes in Mathematics, 1782. Springer-Verlag, Berlin, 2002.

[ER 1 E. Effros and Z.-J. Ruan, Operator spaces, Oxford University Press, 2000.

$\left[\mathrm{ER}_{2}\right] \quad$ E. Effros and Z.-J. Ruan, Operator space tensor products and Hopf convolution algebras J. Operator Theory 50 (2003), 131-156.

[Eno] M. Enock, Measured quantum groupoids in action, Mém. Soc. Math. Fr. (N.S.), no. 114 (2008). 
[EOR] E. G. Effros, N. Ozawa, and Z.-J. Ruan, On injectivity and nuclearity for operator spaces, Duke Math. J. 110 (2001), 489-521.

[Ha 1 M. Hamana, Triple envelopes and Šilov boundaries of operator spaces, Math. J. Toyama Univ. 22 (1999), 77-93.

[Ha $\mathrm{Ha}_{2}$ M. Hamana, Injective envelopes of dynamical systems, Math. J. Toyama Univ. 34 (2011), 23-86.

[Izu] M. Izumi, Non-commutative Poisson boundaries and compact quantum group actions, Adv. Math. 169 (2002), 1-57.

[JNR] M. Junge, M. Neufang and Z.-J. Ruan, A representation theorem for locally compact quantum groups, Int. J. Math. 20 (2009), 377-400.

$\left[\mathrm{KNR}_{1}\right]$ M. Kalantar, M. Neufang and Z.-J. Ruan, Poisson boundaries over locally compact quantum groups, Int. J. Math. 24 (2013), 1350023.

$\left[\mathrm{KNR}_{2}\right]$ M. Kalantar, M. Neufang and Z.-J. Ruan, Realization of quantum group Poisson boundaries as crossed products, Bull. Lond. Math. Soc. 46 (2015), 1267-1275.

[KaS] P. Kasprzak and P. Soltan, Quantum groups with projection on von Neumann algebra level, J. Math. Anal. Appl. 427 (2015), 289-306.

[Kus] J. Kustermans, Locally compact quantum groups in the universal setting, Internat. J. Math. 12 (2001), 289-338.

$[\mathrm{KuV}] \quad$ J. Kustermans and S. Vaes, Locally compact quantum groups Ann. Sci. École Norm. Sup. (4) 33 (2000), 837-934.

[NaT] Y. Nakagami and M. Takesaki, "Duality for Crossed Products of von Neumann Algebras," Lecture Notes in Mathematics, 731. Springer, Berlin-Heidelberg-New York, 1979.

[NeR] M. Neal and B. Russo, Operator space characterizations of $\mathrm{C}^{*}$-algebras and ternary rings, Pacific J. Math. 209 (2003), 339-364.

[NSSS] M. Neufang, P. Salmi, A. Skalski and N. Spronk, Contractive idempotents on locally compact quantum groups, Indiana Univ. Math. J. 62 (2013), 1983-2002.

[SS $\mathrm{SS}_{1} \quad$ P. Salmi and A. Skalski, Idempotent states on locally compact quantum groups, Quart. J. Math. 63 (2012), 1009-1032.

$\left[\mathrm{SS}_{2}\right] \quad$ P. Salmi and A. Skalski, Inclusions of ternary rings of operators and conditional expectations, Math. Proc. Camb. Phil. Soc. 155 (2013), 475-482.

[SkZ] A. Skalski and J. Zacharias, Approximation properties and entropy estimates for crossed products by actions of amenable discrete quantum groups, J. Lond. Math. Soc. (2) 82 (2010), 184-202.

[Sol] B. Solel, Isometries of Hilbert C*-modules, Trans. Amer. Math. Soc. 353 (2001), 4637-4660.

[Str] S. Strătilă, Modular theory in operator algebras, Abacus Press, Tunbridge Wells, 1981.

[Tak] M. Takesaki, Theory of operator algebras. I, Encyclopaedia of Mathematical Sciences, vol. 124, Springer-Verlag, Berlin, 2002.

[You] M. A. Youngson, Completely contractive projections on $C^{*}$-algebras, Quart. J. Math. Oxford Ser. (2) 34 (1983), 507-511.

[Zet] H. H. Zettl, Ideals in Hilbert modules and invariants under strong Morita equivalence of $\mathrm{C}^{*}$-algebras, Arch. Math. 39 (1982), 69-77.

Department of Mathematical Sciences, University of Oulu, PL 3000, Fi-90014 Oulun Yliopisto, FINLAND

E-mail address: pekka.salmi@iki.fi

Institute of Mathematics of the Polish Academy of Sciences, ul. Śniadeckich 8, 00-656 WARszaWA, Poland

E-mail address: a.skalski@impan.pl 